Artigo original

\title{
Perfil dos atendimentos de um pronto socorro hospitalar do Extremo Sul Catarinense
}

\author{
Karina Dal Pont Zilli*, Maria Teresa Brasil Zanini**, Cristiane Damiani Tomazzi, M.Sc.***, \\ Luciana Rosa, M.Sc. ${ }^{* *}$, Karina Gulbis Zimmermann, M.Sc. ${ }^{* *}$, Luciane Bisognin Ceretta, D.Sc. ${ }^{* * *}$
}

*Acadêmica do Curso de Enfermagem da Universidade do Extremo Sul Catarinense - UNESC, **Professora Especialista do Curso de Enfermagem da Universidade do Extremo Sul Catarinense - UNESC, ${ }^{* * *}$ Professora do Curso de Enfermagem da Universidade do Extremo Sul Catarinense - UNESC

\section{Resumo}

Trata-se de um estudo sobre o perfil dos atendimentos de um Pronto Socorro Hospitalar, localizado na regiáo do extremo sul de Santa Catarina (SC) com o objetivo de identificar os atendimentos de acordo às variáveis selecionadas: gênero, faixa etária, os motivos da procura pelo atendimento no pronto socorro, o horário e dias da semana de maior procura destes atendimentos, local de residência, bem como, reconhecer dentre os agravos aqueles que poderiam ter sido atendidos na rede de atenção básica à saúde. Utilizando-se uma pesquisa quantitativa, de cunho documental e descritivo, os dados foram coletados do prontuário eletrônico, com períodos pré-determinados e critérios estabelecidos. A amostra incluiu um total de 12.284 atendimentos nos meses selecionados previamente. Os dados coletados foram inseridos em planilha eletrônica para posterior análise estatística, pelo software estatístico SPSS. Através desta pesquisa, os resultados apontam para fatores e motivos relevantes dos atendimentos no pronto socorro. Conclui-se que a resolubilidade das unidades básicas e secundárias de saúde é condição fundamental para evitar a maioria dos encaminhamentos desnecessários aos centros de complexidade terciária e, particularmente, aos hospitais permitindo que seus leitos sejam ocupados por usuários que realmente deles necessitem.

Palavras-chave: sistema único de saúde, atenção à saúde, atenção primária à saúde, atenção terciária à saúde.

\section{Abstract \\ Profile of the victims of a hospital emergency room of Southern Santa Catarina}

This is a study on the profile of the victims of a Hospital Emergency Room, located in the extreme south of Santa Catarina (SC) in order to identify the calls according to selected variables: gender, age, reasons for demand for care in the emergency room, time and days of week of high demand for these visits, place of residence, and, recognizing among patients those who could have been attended in primary health care. Using a quantitative study of documentary and descriptive method, data were collected from electronic medical records, with pre-determined and established criteria. The sample included a total of 12.284 visits in the months previously selected. The collected data were entered into a spreadsheet for further statistical analysis by SPSS software. Through this research, the results point to important factors 
and reasons of the visits to the emergency room. It follows that the solvability of the basic units and secondary health is a fundamental condition to avoid unnecessary referrals to the most complex tertiary centers, and particularly to hospitals allowing their beds are occupied by users who really need them.

Key-words: unified health system, public health, primary health care, tertiary health care.

\section{Resumen}

\section{Perfil de las víctimas de una sala de emergencias de un hospital del sur de Santa Catarina}

Se trata de un estudio sobre el perfil de las víctimas atendidas en emergencias de un hospital, ubicado en el extremo sur de Santa Catarina (SC) con el fin de identificar la atención de urgencias de acuerdo a las variables seleccionadas: sexo, edad, motivos de demanda de atención en la sala de emergencias, la hora y día de la semana de mayor demanda de esta atención, lugar de residencia, y, reconocer entre los problemas de salud los que podrían haber sido atendidos en la atención primaria de salud. Se utilizó un estudio cuantitativo, documental y descriptivo, cuyos datos se obtuvieron de los registros médicos electrónicos, con criterios pre-determinados y establecidos. La muestra incluyó un total de 12.284 visitas en los meses previamente seleccionados. Los datos obtenidos se introdujeron en el software informático de cálculo SPSS para el análisis estadístico. A través de esta investigación, los resultados apuntan factores importantes y motivos relevantes para demandar asistencia en la sala de emergencias. Se concluye que la solvencia de las unidades básicas de salud y secundarias es una condición fundamental para evitar la mayoría de las derivaciones innecesarias a los centros de atención terciaria complejos, y, en particular, a los hospitales que permitan que sus camas estén ocupadas por los usuarios que realmente las necesitan.

Palabras-clave: sistema único de salud, atención a la salud, atención primaria de salud, atención terciaria de salud

\section{Introdução}

A grande demanda de pacientes no nível terciário de saúde dá uma ideia de que a rede de atenção à saúde não está atuando como deveria, ou que a população encontra maior resolutividade para seus problemas de saúde no hospital ou ainda que desconheçam os serviços ofertados pela rede de atenção à saúde [1-2].

A triagem é utilizada para selecionar os pacientes em grupos com base na gravidade de seus problemas de saúde e imediatismo com o qual esses problemas devem ser tratados [3].

Nas situações de demanda inadequada, ou seja, quando o paciente procura atendimento hospitalar por causas sensíveis a atenção básica, logo após o atendimento da urgência, preconiza-se que seja feito o redirecionamento para a devida inserção no SUS, prosseguindo com o tratamento na rede de atenção primária [1].

Uma das principais diretrizes atuais do $\mathrm{Mi}$ nistério da Saúde é executar a gestão pública com base na indução, monitoramento e avaliação de processos e resultados mensuráveis, garantindo acesso e qualidade da atenção em saúde a toda a população. Diversificam-se os esforços para ajustar as estratégias previstas na Política Nacional de Atenção Básica (PNAB) na direção de reconhecer a qualidade dos serviços de Atenção Básica (AB) ofertados à sociedade [4].

A oferta adequada de atenção primária à saúde é fator relevante para evitar atendimento hospitalar por condiçóes sensíveis à atenção ambulatorial. Há necessidade de organizar a rede de cuidados de modo a melhorar a articulação entre diferentes esferas de atenção [5].

A ideia básica da organização do sistema de saúde é fazer com que o usuário entre pela atenção primária e aquilo que náo se consegue resolver nesse nível seja encaminhado para o secundário, que por sua vez irá referir para o setor terciário situaçóes as quais não esteja capacitada a atender [4].

O PMAQ (Programa de Melhoria de Acesso e Qualidade da Atenção Básica) foi instituído pela Portaria de no 1.654 GM/MS do dia 19 de julho de 2011 e foi produto de um importante processo de negociação e pactuação das três esferas de gestão do SUS criado como um instrumento norteador na 
rede de atençáo. O principal objetivo do Programa é induzir a ampliaçáo do acesso e a melhoria da qualidade da atenção básica, com garantia de um padrão de qualidade comparável nacional, regional e localmente de maneira a permitir maior transparência e efetividade das açóes governamentais direcionadas à Atenção Básica em Saúde [6].

Diante deste contexto, o estudo identificou o perfil dos atendimentos de um pronto socorro hospitalar de um município do Extremo Sul Catarinense, buscando elencar os atendimentos sensíveis à atenção básica, contribuindo com a organização do sistema, bem como com o processo de produção de saúde.

\section{Material e métodos}

A abordagem metodológica adotada para este estudo foi quantitativa, descritiva documental, uma vez que foi embasado na análise dos dados contidos no prontuário eletrônico dos pacientes atendidos nos meses de fevereiro, maio, agosto e novembro de 2011 no pronto socorro de um hospital de Santa Catarina, extraindo os dados necessários para pesquisa e enviados para planilha do Excel para posterior análise. A amostra incluiu um total de 12.284 atendimentos nos meses selecionados.

O estudo foi aprovado pelo Comitê de Ética em Pesquisa, conforme a Resolução 196/96 do Conselho Nacional de Saúde (CNS) sob o protocolo $10.210 / 2012$, e obteve autorização do gerente do campo de estudo.

Os dados encontrados nos prontuários eletrônicos foram transformados em relatórios estatísticos. De acordo com estas informaçóes foi elaborada uma planilha eletrônica, no formato Microsoft Excel, com todos os atendimentos realizados nos meses mencionados anteriormente. As variáveis de interesse, neste estudo, foram: faixa etária, gênero, município, bairro, motivo, horário e dias da semana de atendimento.

Os dados armazenados em planilha eletrônica foram transportados para o software estatístico SPSS (Statistical Package for the Social Science, SPSS Inc., Chicago) versão 17.0 para Windows, procedendo à análise estatística dos dados descritivos, apresentados na forma de frequência e percentual.

\section{Resultados}

A Tabela I apresenta a distribuição dos atendimentos por gênero nos meses de fevereiro, maio, agosto e novembro de 2011, onde se pode observar que o gênero feminino prevaleceu durante os meses de maio e agosto com 50,55\%.

$\mathrm{Na}$ variável faixa etária identificou-se que o maior número de atendimentos foi na idade de 25 a 30 anos, representando $9,88 \%$ do total de atendimentos, seguido pela faixa etária de 0 a 5 anos que apresentou $9,62 \%$ e a idade de 30 a 35 anos com $9,17 \%$ dos atendimentos.

Tabela I - Distribuição dos atendimentos de acordo com a faixa etária e gênero, nos meses de fevereiro, maio, agosto e novembro de 2011 no pronto socorro.

\begin{tabular}{lcc}
\hline \multicolumn{1}{c}{ Faixa etária } & $\mathrm{N}$ & $\%$ \\
\hline 0 a 5 & 1182 & 9,62 \\
5 a 10 & 655 & 5,33 \\
10 a 15 & 590 & 4,80 \\
15 a 20 & 920 & 7,49 \\
20 a 25 & 920 & 7,49 \\
25 a 30 & 1214 & 9,88 \\
30 a 35 & 1127 & 9,17 \\
35 a 40 & 756 & 6,15 \\
40 a 45 & 808 & 6,58 \\
45 a 50 & 803 & 6,54 \\
50 a 55 & 736 & 5,99 \\
55 a 60 & 690 & 5,62 \\
60 a 65 & 550 & 4,48 \\
65 a 70 & 449 & 3,66 \\
70 e + & 884 & 7,20 \\
Total & 12284 & 100,00 \\
\hline \multicolumn{2}{c}{ Gênero } & 6209 \\
\hline Feminino & 6075 & 50,55 \\
Masculino & 12284 & 100,45 \\
Total & &
\end{tabular}

Os resultados na Tabela II mostram que $70,14 \%$ dos atendimentos dos pacientes possuem procedência do município de Araranguá sede da Instituição hospitalar, evidenciando fatores relativos à regionalização dos serviços. No segundo momento está o município de Arroio do Silva, distante $7,8 \mathrm{~km}$ do hospital com 13,20\% e o município Maracajá com $5,31 \%$, que se situa a $15,8 \mathrm{~km}$ do hospital.

No que se refere aos bairros observa-se que o bairro Coloninha apresentou 11,34\% dos atendimentos, seguido pelo bairro centro com $9,16 \%$ e o bairro Mato alto com 6,06\%. Justifica-se este resultado considerando a proximidade destes bairros com a Instituição hospitalar como também o acesso aos serviços de complexidade diferenciada da atenção básica. Convém destacar que o bairro Jardim das 
Avenidas faz divisa com o bairro Coloninha, portanto bem mais próximo do hospital de estudo do que o bairro centro, isso posto seria de se esperar que houvesse maior número de atendimento deste bairro do que do bairro centro. No entanto, observou-se que o bairro centro apresentou a segunda maior frequência de procura ao atendimento.

Tabela II - Distribuição dos atendimentos de acordo com os municípios e bairros nos meses de fevereiro, maio, agosto e novembro de 2011 no pronto socorro.

\begin{tabular}{|c|c|c|}
\hline Variáveis & $\mathrm{N}$ & $\%$ \\
\hline \multicolumn{3}{|l|}{ Municípios } \\
\hline Araranguá & 8616 & 70,14 \\
\hline Balneário Arroio do Silva & 1622 & 13,20 \\
\hline Maracaja & 652 & 5,31 \\
\hline Sombrio & 363 & 2,96 \\
\hline Criciúma & 84 & 0,68 \\
\hline Balneário Gaivota & 95 & 0,77 \\
\hline Ermo & 69 & 0,56 \\
\hline Turvo & 92 & 0,75 \\
\hline Meleiro & 96 & 0,78 \\
\hline Jacinto Machado & 75 & 0,61 \\
\hline Santa Rosa Do Sul & 87 & 0,71 \\
\hline Outros & 433 & 3,52 \\
\hline Total & 12284 & 100,00 \\
\hline \multicolumn{3}{|l|}{ Bairros } \\
\hline Coloninha & 1393 & 11,34 \\
\hline Centro & 1125 & 9,16 \\
\hline Urussanguinha & 682 & 5,55 \\
\hline Mato Alto & 745 & 6,06 \\
\hline Lagoão & 598 & 4,87 \\
\hline Jardim Avenidas & 664 & 5,41 \\
\hline Policia Rodoviária. & 526 & 4,28 \\
\hline Cidade Alta & 418 & 3,40 \\
\hline Jardim Cibele & 330 & 2,69 \\
\hline Vila São José & 300 & 2,44 \\
\hline Divinéia & 307 & 2,50 \\
\hline Outros & 5196 & 42,30 \\
\hline Total & 12284 & 100,00 \\
\hline
\end{tabular}

A Tabela III demonstra o período do dia com maior número de atendimentos, sendo que no período matutino o horário das 06 às 11 horas apresenta $28,53 \%$. No período noturno das 18 às 24 horas o percentual de atendimentos foi de $30,23 \%$, já no período da 0 às 5 horas foi de $9,8 \%$.

Quando analisados os atendimentos por períodos de 12 horas, verificou-se que no horário das 12 às 24 horas ocorreram $61,63 \%$ dos atendimentos, contrapondo com $38,37 \%$ no período da 0 às 12 horas.
Em relaçáo ao dia da semana que mais houve atendimento quando considerado a totalidade de atendimentos foi à segunda-feira com 16,7\% comparando com o menor número que foi no sábado com $12,4 \%$ de atendimentos.

Tabela III - Distribuição dos atendimentos de acordo com o horário e dias da semana nos meses de fevereiro, maio, agosto e novembro de 2011 no pronto socorro.

\begin{tabular}{lcc}
\hline \multicolumn{1}{c}{ Variáveis } & $\mathrm{N}$ & $\%$ \\
\hline \multicolumn{1}{c}{ Horário } & \\
\hline O0h00 às $5 \mathrm{~h} 00$ & 1209 & 9,84 \\
$06 \mathrm{~h} 00$ às $11 \mathrm{~h} 00$ & 3505 & 28,53 \\
$12 \mathrm{~h} 00$ às $17 \mathrm{~h} 00$ & 3857 & 31,40 \\
$18 \mathrm{~h} 00$ às $24 \mathrm{~h} 00$ & 3713 & 30,23 \\
Total & 12284 & 100,00 \\
\hline \multicolumn{1}{c}{ Dias da Semana } & & \\
\hline Segunda-Feira & 2031 & 16,53 \\
Terça-Feira & 1965 & 16,00 \\
Quarta-Feira & 1843 & 15,00 \\
Quinta-Feira & 1627 & 13,24 \\
Sexta-Feira & 1718 & 13,99 \\
Sábado & 1530 & 12,46 \\
Domingo & 1570 & 12,78 \\
Total & 12284 & 100,00 \\
\hline
\end{tabular}

Os motivos de atendimento apresentados na Tabela IV denotam a dor aguda e crônica como uma das causas que mais direcionam os usuários ao pronto socorro com um percentual de $18,41 \%$, seguida das afecçôes esqueléticas em 14,43\%, afecçôes do trato respiratório com $12,72 \%$, trato gastrointestinal com $8,78 \%$, seguido do trauma com $8,69 \%$ e motivos não especificados no prontuário eletrônico com um percentual de $10,67 \%$.

A Tabela $\mathrm{V}$ demonstra que dentre os atendimentos realizados no pronto socorro, os motivos sensíveis à rede de Atenção Básica, destacam-se a dor lombar com 474 atendimentos, seguida da hipertensão arterial com 300, diarreia com 255 e cefaleia com 222 atendimentos.

\section{Discussão}

Historicamente a população feminina procura mais por atendimentos do que o gênero masculino, porém, evidenciou-se que houve uma frequência de dois meses distintos por procura ao atendimento de ambos os gêneros. Nos meses de fevereiro e novembro prevaleceu o atendimento no gênero masculino com $40,45 \%$ proporcionalmente ao 
Tabela IV - Distribuição dos motivos de atendimento nos meses de fevereiro, maio, agosto e novembro de 2011 no pronto socorro.

\begin{tabular}{lcccccc}
\hline \multicolumn{1}{c}{ Motivo do Atendimento } & Fevereiro & Maio & Agosto & Novembro & $\mathrm{N}$ & $\%$ \\
\hline Acidentes Animais Peçonhentos & 11 & 9 & 9 & 21 & 50 & 0,41 \\
Afecções Aparelho Reprodutor & 26 & 16 & 24 & 24 & 90 & 0,73 \\
Afecções do Sistema Respiratório & 377 & 389 & 395 & 402 & 1563 & 12,72 \\
Afecções do Sistema Urinário & 190 & 153 & 135 & 184 & 662 & 5,39 \\
Afecções do TGl & 421 & 202 & 184 & 272 & 1079 & 8,78 \\
Afecções Sistema Circulatório & 180 & 247 & 225 & 245 & 897 & 7,30 \\
Afecções Sistema Esquelético & 494 & 407 & 382 & 489 & 1772 & 14,43 \\
Afecções Sistema Nervoso & 55 & 32 & 49 & 52 & 188 & 1,53 \\
Álcool e Outras Drogas & 15 & 15 & 16 & 26 & 72 & 0,59 \\
Discrasias Sanguíneas & 3 & 1 & 5 & 3 & 12 & 0,10 \\
Dor Aguda e Crônica & 607 & 542 & 527 & 586 & 2262 & 18,41 \\
Febre & 85 & 36 & 32 & 39 & 192 & 1,56 \\
Infecções e Inflamações & 82 & 269 & 284 & 155 & 790 & 6,43 \\
Não Especificado & 271 & 367 & 376 & 297 & 1311 & 10,67 \\
Neoplasias & 2 & 4 & 3 & 6 & 15 & 0,12 \\
Transtornos Mentais & 77 & 54 & 58 & 73 & 262 & 2,13 \\
Trauma e Ferimentos & 274 & 237 & 223 & 333 & 1067 & 8,69 \\
Total & & & & & 12284 & 100,00 \\
\hline
\end{tabular}

Tabela V - Distribuição dos motivos de atendimento sensíveis a Atenção Básica nos meses de fevereiro, maio, agosto e novembro de 2011 no pronto socorro.

\begin{tabular}{lc}
\hline Atendimentos para a Atenção Básica & $\mathrm{N}$ \\
\hline Amidalite & 18 \\
Anemias & 8 \\
Candidíase & 5 \\
Cefaleia & 222 \\
Dermatite & 119 \\
Desnutrição & 4 \\
Diarreia & 255 \\
Dor Lombar & 474 \\
Dor Muscular & 83 \\
Enxaqueca & 32 \\
Herpes Zoster & 5 \\
Hipertensão & 300 \\
Infecção do Ouvido & 53 \\
Infecções Urinárias & 92 \\
Parotidite & 2 \\
Rubéola & 1 \\
Úlcera Varicosa & 17 \\
Varicela & 9 \\
Total & 1699 \\
\hline
\end{tabular}

total dos atendimentos. Isto leva a supor que talvez seja relacionado ao maior número da população flutuante devido aos meses com temperaturas mais elevadas, acarretando, assim, maior fluxo de veículos automotivos nas rodovias federais e estaduais por ser o município de referência uma cidade que possui praias com belezas naturais. Estudos evidenciam variação nos meses do ano, e supôe que tal situação pode decorrer de variaçóes sazonais e regionais que interferem no padrão de adoecimento. Isso pode consequentemente acarretar no aumento da procura pelo serviço [4].

Identificar a idade dos pacientes atendidos permite caracterizar o atendimento ou a fragilidade da rede de atenção, quando se verificam situações de atendimento de problemas de saúde que poderiam ter sido resolvidos na atenção básica, conforme os princípios do SUS. Além disso, a faixa etária menor de 05 anos e adultos jovens estáo contemplados na maioria dos programas de saúde da atenção básica, não se justificando entáo a procura de atendimento hospitalar por problemas não urgentes e emergentes [7].

A frequência apresentada pela procura no atendimento por outros municípios também pode ser explicada devido à complexidade do hospital, uma vez que a magnitude dos recursos materiais e humanos disponíveis neste serviço pode servir de referência para casos que necessitem de atenção especializada, para pacientes de áreas que extrapolem os limites do município [4].

O acesso é um atributo dos serviços de saúde bastante valorizado pelos usuários, populaçóes estas que vivem em condições socioeconômicas precárias e que buscam os serviços de forma assistencial, interessado mais por um padrão mínimo de sobrevivência do que pela própria qualidade dos serviços [8]. 
Quando analisados os atendimentos por períodos de 12 horas, verificou-se que no horário das 12 às 24 horas ocorreram $61,63 \%$ dos atendimentos, contrapondo com 38,37\% no período da 0 às 12 horas. Dados encontrados na literatura associam o período das 12 às 24 horas, onde ocorreram $67,1 \%$ dos atendimentos. Cabe destacar que há relatos de serviços de urgência e emergência nos quais 65\% dos casos atendidos não constituem emergência verdadeira [5-9].

No que diz respeito aos motivos de atendimento a dor ficou em maior evidência conforme os resultados apresentados, e está presente em mais de 70\% dos pacientes que buscam os consultórios brasileiros por motivos diversos, sendo a razão de consultas médicas em um terço dos casos. A atenção básica é de extrema importância neste motivo de busca ao pronto atendimento, pois, vários programas nesta área da saúde estão à disposição aos usuários do SUS. Neste sentido, seria importante colocar no prontuário eletrônico o tipo de dor referida pelo paciente assim como também a localização [10].

Em relação aos distúrbios esqueléticos que obteve resultados importantes na pesquisa, deve-se considerar a qualidade de vida que as pessoas na atualidade possuem, pois, a maior parte náo realiza exercícios físicos semanais, a alimentaçáo devido ao estresse diário não está de maneira equilibrada, bem como o sono e repouso não é de boa qualidade. Estudos abordam que as afecçôes referentes aos distúrbios musculoesqueléticos também são causadas em sua grande maioria por acidentes de trânsito. Esses determinam uma série de prejuízos individuais e para a sociedade. $\mathrm{O}$ trauma de acidentes de motocicleta tem poder destrutivo com crescente incidência na vida moderna [11-12].

As afecçóes do sistema respiratório aparecem no estudo com destaque importante também. $\mathrm{O}$ aumento da incidência de doenças do aparelho respiratório é decorrente da intensificação da poluição atmosférica, sendo estes efeitos tanto agudos como crônicos [13].

Observa-se que a frequência de atendimentos sensíveis à atenção básica não é tão expressiva, porém, devem-se considerar os motivos não especificados no sistema do prontuário eletrônico, neste caso aparece com 10,67\% dos registros, assim como os motivos de atendimentos que mais foram expressivos da frequência total, citando a dor aguda e crônica, afecçóes esqueléticas e afecçóes respiratórias que não foram encontradas relaçôes mais específicas destes motivos. Logo, alguns destes atendimentos poderiam estar direcionados também para a atenção primária à saúde [6].

Encontrou-se na pesquisa a hipertensão como um motivo bastante evidente de procura ao pronto atendimento. Destaca-se a oferta na rede de atenção básica do Programa Hiperdia, com todas as ferramentas disponíveis para os profissionais e usuários estarem inseridos no processo de produção de saúde. Questiona-se em qual nível da atenção primária estão ocorrendo nós críticos para que estes usuários procurem o pronto socorro com crises hipertensivas [6-14].

A dor lombar inserida dentre os motivos sensíveis a Atenção Básica aparece com ênfase. Destaca-se aqui o Programa Academia da Saúde criado pela Portaria Ministerial n ${ }^{\circ} 719$ de abril de 2011, que tem como objetivo principal contribuir para a promoção da saúde da população a partir da implantação de polos com infraestrutura, equipamentos e quadro de pessoal qualificado para a orientação de práticas corporais e atividade física e modos de vida saudáveis [11]

Acredita-se que seja necessário um melhor planejamento e comprometimento por parte dos gestores para atender as necessidades da populaçáo com qualidade, pois, o SUS é o mesmo para todos e em alguns municípios é mais bem estruturado do que em outros. Há também que se considerar a questão cultural dos usuários, porque, às vezes, mesmo tendo à disposição uma rede de atenção estruturada acabam buscando o serviço do pronto socorro por melhor acessibilidade aos serviços de média e alta complexidade $[6,14,15]$.

\section{Conclusão}

Com o aumento da demanda nos serviços de urgência e emergência hospitalar, a maior parte das Instituiçôes de saúde necessita reorganizar seus serviços para continuar mantendo um serviço de qualidade e que atenda as necessidades dos usuários.

Conclui-se que um número de atendimentos no pronto socorro ocorre dentro do horário em que a atenção básica disponibiliza os serviços de saúde. Os motivos que levaram os usuários ao pronto socorro também chamam a atenção da pesquisa, pois, uma parte importante seria para resolubilidade da atenção básica. Neste ínterim cabe ressaltar que a falha na alimentação dos dados no sistema do prontuário eletrônico também influenciou nos resultados 
encontrados, pois, alguns motivos foram, desta forma, englobados em atendimentos de urgência e emergência. Por isso, encontraram-se resultados sensíveis para a atenção básica com menor frequência do que o esperado nos pressupostos. Porém, estes nós críticos no sistema de retroalimentação do software são fatores preponderantes que influenciaram nos resultados do estudo. Outro elemento influenciador nos resultados refere-se à maneira que o usuário coloca a queixa principal na porta de entrada do pronto socorro. Muitas vezes os usuários por causas diversas e cognitivas acabam exacerbando o motivo principal para atendimento, com o intuito de receber o acolhimento devido no serviço de referência hospitalar. Onde podemos rever estas questóes? Em qual momento da atenção primária o usuário perde-se em um caminho que muitas vezes não ocorre o retorno, tornando a busca ao pronto socorro hospitalar uma ferramenta principal de produção de saúde?

Torna-se importante consolidar o desafio de melhor estruturar a rede de atenção através da Estratégia Saúde da Família, implantada nas Unidades Básicas de Saúde da rede municipal, para facilitar o acesso e garantir a resolutividade dos agravos sensíveis a atenção básica a fim de por em pratica as diretrizes do PMAQ e, consequentemente, garantir assistência e desafogar os serviços de emergência.

A resolubilidade das unidades básicas e secundárias de saúde é condição fundamental para evitar a maioria dos encaminhamentos desnecessários aos centros de complexidade terciária e, particularmente, aos hospitais permitindo que seus leitos sejam ocupados por usuários que realmente deles necessitem.

\section{Referências}

1. Santos NCM. Urgência e Emergência para enfermagem: do atendimento pré-hospitalar APH à sala de emergência. 5a ed. São Paulo: Iátria; 2008.

2. Figueiredo NMA. Enfermagem: Cuidando em Emergência. São Paulo: Yendis; 2005.

3. Smeltzer SC, Bare BG. Brunner \& Suddarth: Tratado de Enfermagem Médico Cirúrgico. 9a ed. Rio de Janeiro: Guanabara Koogan; 2002. p.37.

4. Ministério da Saúde. Portaria 2.488 de 21 de outubro de 2011. Aprova a Política Nacional de Atenção Básica. Brasília: MS; 2011.

5. Coelho MF, Chaves LDP, Anselmi ML, Hayashida M, Santos CB. Análise dos aspectos organizacionais de um serviço de urgências clínicas: estudo em um hospital geral do município de Ribeiráo Preto, SP, Brasil. Rev Latinoam Enferm 2010;18(4):770-77.

6. Ministério da Saúde. Portaria 1.654 de 19 de julho de 2011. Institui no Sistema Único de Sáude, o Programa Nacional de Melhoria do Acesso e da Qualidade da Atenção Básica (PMAQ-AB). Brasília: MS; 2011.

7. Travassos C, Martins M. Uma revisão sobre os conceitos de acesso e utilizaçáo de serviços de saúde. Cad Saúde Pública 2004;20(Supl.2):190-8.

8. Esperidião $M$, Leny $A B$. Avaliação de satisfação de usuários. Ciênc Saúde Coletiva 2005;10:303-12.

9. Souza JB. Poderia a atividade física induzir analgesia em pacientes com dor crônica?. Revista Brasileira de Medicina do Esporte 2009;15(2):145-50.

10. Rocha APC. Dor: aspectos atuais da sensibilização periférica e central. Revista Brasileira de Anestesiologia 2007;57(1):94-105.

11. Magnago TSBS, Lisboa MTL, Souza IEO, Moreira MC. Distúrbios músculo-esqueléticos em trabalhadores de enfermagem: associação com condiçóes de trabalho. Rev Bras Enferm 2007;60(6):701-5.

12. Dubeux LS, Freese E, Reis YAC. Avaliação dos serviços de urgência e emergência da rede hospitalar de referência no Nordeste Brasileiro. Cad Saúde Pública 2010;26(8):1508-18.

13. Hess SC. Distribuição Espacial da Mortalidade por Doenças do Aparelho Respiratório no Brasil. Engenharia Ambiental - Espírito Santo do Pinhal 2009; 6(3):607-624.

14. Ministério da Saúde. Portaria 399 de 27 de fevereiro de 2006. Institui as diretrizes Operacionais - Pactos pela Vida, em Defesa do SUS e de Gestão. Brasília: MS; 2006.

15. Campozana GG. Satisfação dos usuários com a assistência de saúde no estado de Pernambuco. Ciênc Saúde Coletiva 2011;16(3):1849-61. 